\title{
Salivary steroids and psychometric parameters in male marathon runners
}

\author{
B. Harris, MRCPsych ${ }^{1}$, N.J. Cook, BSc ${ }^{2}$, R.F. Walker, $\mathrm{PhD}^{2}$, G.F. Read, $\mathrm{PhD}^{2}$ and \\ D. Riad-Fahmy, $\mathbf{P h D}^{2}$
}

\begin{abstract}
Summary
Testosterone and cortisol in male marathon runners $(\mathbf{n}=11)$ were determined in saliva samples $(n=28)$ collected during the three rest days preceding a competitive marathon and in the samples collected at $08.00 \mathrm{~h}$ on the race day. An Eysenck Personality Inventory was completed on the first rest day and psychological state was assessed on rest days and on the morning of the marathon by completion of visual analogue scales for anxiety, depression, hostility and libido at four times each day. Anxiety, depression and hostility were positively inter-correlated. Extraversion and depression were negatively correlated. At $08.00 \mathrm{~h}$ on the day of the marathon, anxiety and hostility scores were significantly higher than those on rest days, but depression and libido scores were unchanged. No relationship was found between depression or libido and any hormonal parameter. Race day cortisol correlated negatively with hostility, and changes in cortisol $(09.00 \mathrm{~h})$ between the race day and the mean restday levels correlated with the corresponding changes in anxiety.
\end{abstract}

Keywords: Salivary steroids, psychometric indices, marathon runners

\section{Introduction}

As marathon running is a popular recreational activity, attention has been directed in this pilot study towards the introduction of techniques better suited to assessing the effect of strenuous exertion on endocrine function and on changes in mood. The runners' 'high' is well described as is also a feeling of 'well being' after running. This has been shown to be associated with plasma endorphin levels ${ }^{1}$. Exercise is also advocated as an effective therapy for chronic depression ${ }^{2}$, and it may well be that such increased endorphinergic activity contributes to the efficacy of this treatment.

Surprisingly little, however, is known concerning the relationship between mental state and stress hor-

\footnotetext{
${ }^{1}$ Department of Psychological Medicine, University of Wales College of Medicine, Cardiff

${ }^{2}$ Steroid Assay Laboratory, Tenovus Institute, University of Wales College of Medicine, Cardiff

Address for correspondence: Dr G.F. Read, Steroid Assay Laboratory, Tenovus Institute, University of Wales College of Medicine, Heath Park, Cardiff
}

(C) 1989 Butterworth \& Co (Publishers) Ltd 0306-3674/89/020089-05 \$03.00 mones in runners prior to and during such demanding competitive events. Recent reports indicate that salivary cortisol and testosterone concentrations provide a reliable index of adrenal and testicular activity ${ }^{3,4}$. Difficulties can occur in obtaining plasma samples prior to a race ${ }^{5}$, but the easy non-invasive collection of saliva has, in the present study, allowed sampling at frequent intervals not only while the runners are resting for the three days prior to the race, but also just prior to the marathon and throughout the course of the run itself.

We have previously reported salivary cortisol and testosterone concentrations before, during and after the marathon in the subjects of the present study ${ }^{6,7}$. These data are reproduced in Figure 1.

On the day of the marathon, mean salivary cortisol $\left(23.5 \mathrm{nmol} \mathrm{L}^{-1}\right)$ and testosterone $\left(258 \mathrm{pmol} \mathrm{L}^{-1}\right)$ concentrations at 08.00 hours showed no significant increase above similarly timed samples on rest days. In samples collected at 09.00 hours, immediately prior to the marathon, cortisol concentrations $(25.1 \mathrm{nmol}$ $\left.\mathrm{L}^{-1}\right)$ were increased $(P<0.05)$, whereas those of testosterone were not. In runners who provided saliva samples throughout the marathon $(n=8)$, concentrations of both steroids rose. Testosterone concentrations (442 pmol $\mathrm{L}^{-1}$ ) were maximal at $21 \mathrm{~m}$, values at this point being 130 per cent above similarly timed samples on rest days. Cortisol concentrations were also increased by 373 per cent. Maximal values $\left(87.9 \mathrm{nmol} \mathrm{L}^{-1}\right.$ ) were observed shortly after completion of the run. Within four hours of completing the marathon, concentrations of both steroids did not differ significantly from those of corresponding rest day values.

Reports of increased adrenal activity in marathon runners just prior to the event have, in the past, been presumed to be due to 'psychological stress ${ }^{8}$. However, data derived from objective measurement of mood states and personality traits are lacking. Apart from factors such as these it is also possible that, in a group activity such as marathon running, social stresses are of importance.

It has been shown that when talapoin monkeys of either sex are caged together, they form themselves into the expected rank order of aggression/submission, and that the most aggressive are sexually dominant'. Subservient males have higher cortisol and prolactin levels, but low testosterone. Similar findings have been reported in a group of five men confined on a 38 foot boat for a two-week sailing holiday ${ }^{10}$. 


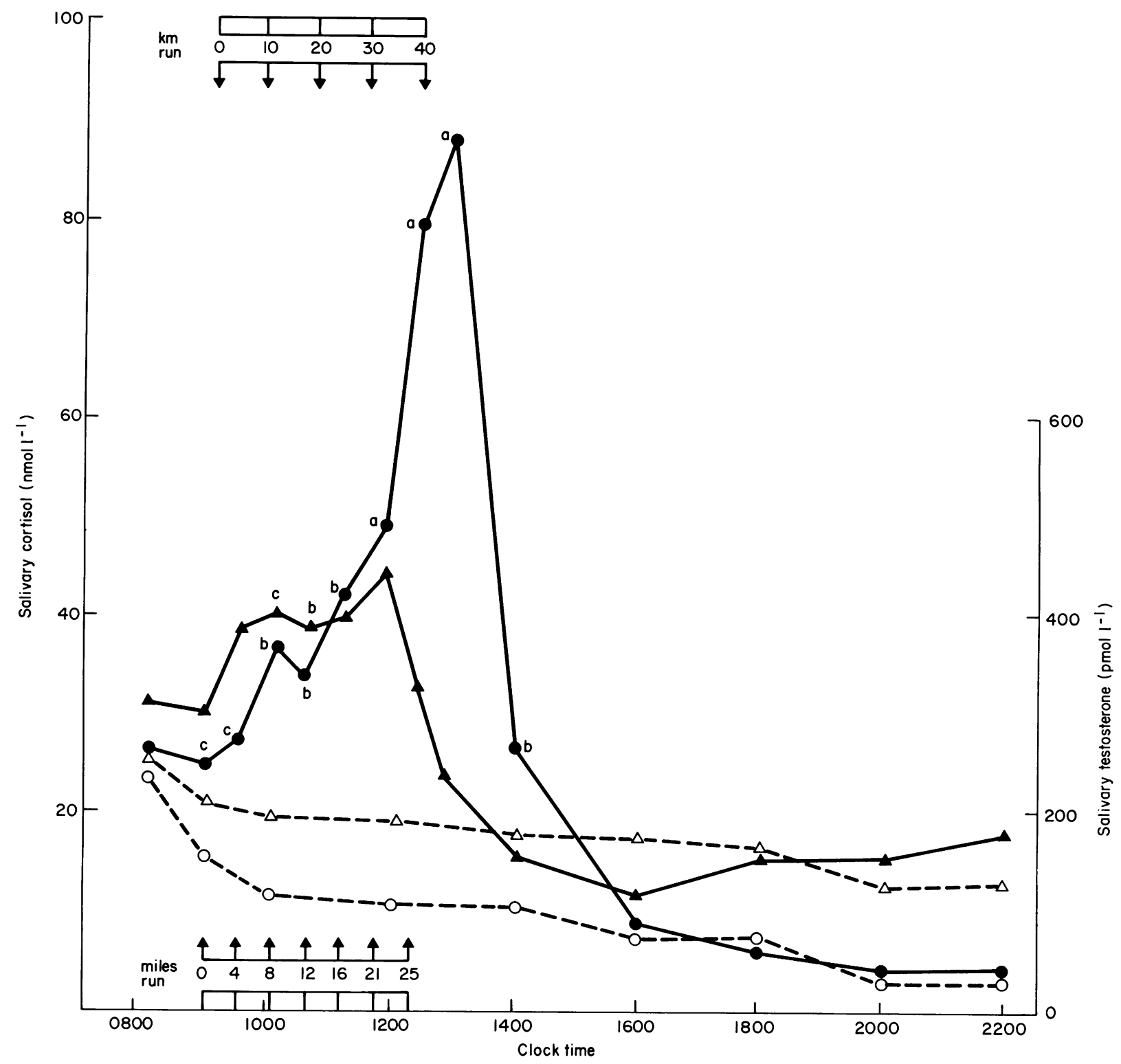

Figure 1. Mean salivary cortisol and testosterone concentrations in eight runners during the Cardiff marathon and on rest days $(-\boldsymbol{\Delta}-)$ salivary testosterone: run day

(-) -) salivary cortisol: run day

$(-\triangle-)$ salivary testosterone: rest day

(-O-) salivary cortisol: rest day

Significance of differences from corresponding rest day values: $\mathrm{a}<0.001 ; \mathrm{b} 0.001<\mathrm{P}<0.01 ; \mathrm{c} 0.01<\mathrm{P}<0.05$

(Taken from Cook et al. ${ }^{6}$ )

Although in a marathon run, the group activity covers only a relatively short period of time, it might be argued that 'conditioning of the mind' begins well in advance of the race itself with the clear identification of elite runners. Furthermore, despite the spatial separation which occurs during the marathon, it remains a group activity since runners pace themselves, in part, by relative distance from other participants.

Thus the possible interrelationships between hormonal and psychological indices of mood in marathon runners was of interest. Whilst monitoring changes in mood and endocrine function, both before and after the marathon, are worthwhile objectives, it was thought advisable in this pilot study to terminate collection of psychological data at the commencement of the run. Logistical considerations suggested that collection of data sheets, indicating mood change during rest days and just prior to the marathon, would be simplified and high compliance rates achieved by selection of a fixed point in time where all participants would be present.

\section{Methods}

Of 15 normal male recruits who participated in the Cardiff marathon, four were hospital staff, whereas the other 11 were from local running clubs. All participants were drug-free, had a mean age of 34.7 years (7.1 SD) and had participated in at least one previous marathon. All had been in training for at least two months prior to the marathon. Training schedules var- 
ied. The maximum distance run in training ranged from 35 to 70 miles per week. All trained well enough to complete the marathon, but none had expectations of winning. Lack of compliance in completion of questionnaires or in the collection of saliva caused four subjects to be omitted from this study.

Participants were instructed how to collect saliva (by allowing it to collect in the floor of the mouth and then gently spitting it out into wide-necked screw-capped tubes of $5 \mathrm{ml}$ capacity). Samples were collected nine times a day at fixed times $(08.00,09.00,10.00,12.00$, $14.00,16.00,18.00,20.00$ and 22.00 hours) on each of the three rest days preceding the marathon. On the day of the marathon, samples were collected at 0800 hours and again just prior to commencement of the marathon at 0900 hours. Although, during the marathon, sampling was based on distance run rather than time, after completion of the run the same sampling regimen as on rest days was resumed.

To facilitate collection and storage of the samples, participants were provided with a box containing the requisite numbers of tubes. As soon as practicable after collection, samples were deep-frozen at $-20^{\circ} \mathrm{C}$.

\section{Assay procedure}

Cortisol and testosterone concentration in saliva samples were determined by in-house radioimmunoassays. In brief, the procedure for cortisol was direct ${ }^{11}$, that for testosterone required solvent extraction ${ }^{4}$. Anti-cortisol sera were raised against the immunogen prepared from the same derivative (cortisol-3-OCMO) as that used for iodination and coupled to a magnetisable solid-phase antiserum. That for testosterone used a $\left[{ }^{3} \mathrm{H}\right]$-radioligand, and required dextran-coated charcoal for separating the antibody-bound and free fractions.

Within and between assay coefficients of variation for high, medium and low quality control samples for cortisol $\left(29.3,11.8\right.$ and $\left.3.0 \mathrm{nmol} \mathrm{L}^{-1}\right)$ did not exceed 11 per cent and 13 per cent. The coefficients of variation for high and low quality control samples for testosterone ( 466 and $310 \mathrm{pmol} \mathrm{L}^{-1}$ ) did not exceed 8 per cent and 9 per cent. The precision profile, constructed according to Ekins ${ }^{12}$, indicated that these assays had lower limits of sensitivity of $0.5 \mathrm{nmol} \mathrm{L}^{-1}$ and $9 \mathrm{pmol}$ $\mathrm{L}^{-1}$ respectively. Measurement of cortisol and testosterone therefore required $50 \mu \mathrm{L}$ and $200 \mu \mathrm{L}$ aliquots of saliva respectively. All samples were processed for both cortisol and testosterone. Mean rest day values were calculated, as also were associated mean hormone ranges. The daily range was taken as the difference between the highest and lowest values for an individual on a given day, and the mean hormone range as the group mean of these individual ranges on that day.

\section{Psychometric indices}

On the three days prior to the run, and on the morning of the run, well accepted visual analogue scales were completed at $08.00,12.00,18.00$ and 22.00 hours in order to monitor mood. These were 10-centimetre lines representing anxiety, depression, hostility ${ }^{13}$, and libido $^{14}$. The subject marks the line at the point which represents his mood at that time. The mid-point of the line represented 'normal' for the subject, and extreme ends represented 'very anxious - calm and peaceful', 'sad and unhappy-joyful and happy',-'angry and aggressive - friendly', 'no sexy thoughts - extreme sexy thoughts'. The Eysenck Personality Inventory was also completed by each subject on the first of the three rest-days ${ }^{15}$.

The associations between hormonal concentrations and psychometric indices were assessed using the Pearson correlation matrix and its non-parametric equivalent, the Spearman rank correlation matrix.

\section{Results and discussion}

This communication describes initial attempts to examine possible interrelationships between changes in mood and endocrine function in the period leading up to a marathon. Endocrine function was assessed by salivary sampling, since this caused no pain or apprehension. The negligible disturbance in the life style or competitive ability of the runners caused by salivary sampling led to the ready acceptance of this technique for investigating endocrine activity. Acceptance of the visual analogue scales, used to assess changes in mood, was also good, and a number of significant associations between psychometric indices were demonstrated. States of anxiety, depression and hostility were all positively associated (Table I). Since these associations are expected, the data provide evidence for the validity of the visual analogue scales. Libido, defined here as tendency towards sexy thoughts, was negatively associated with hostility $(r=-0.54)$. Libido and depression were also negatively associated, but this association did not reach significance $(r=-0.44)$. It is also of interest that extraversion, a 'trait', was associated negatively with the 'states' of hostility and depression.

There were no significant differences in anxiety, hostility, depression and libido between the three rest days. However, the anxiety score at 08.00 hours on the day of the marathon $(70.6 \pm 9.1 ; n=11)$ was significantly greater than $(P<0.0001)$ the mean value of scores obtained at comparable times on rest days $(44.6 \pm 18.4 ; n=33)$. Hostility scores at 08.00 hours on the day of the marathon $(50.1 \pm 21.4 ; n=11)$ were also significantly higher $(P<0.05)$ than mean values on rest days $(37.1 \pm 17.3 ; n=33)$. By contrast, depression and libido scores $(43.6 \pm 13.3 ; 38.8 \pm 21.4)$ did not differ significantly from those obtained on rest days.

Associations were also sought between psychometric indices and hormonal parameters (Tables 2 and 3 ). In these athletes, neither the psychological states of anxiety, depression and libido nor the traits of extraversion and neuroticism was associated with any hormonal parameter. There was, however, a significant negative correlation between 'race day 08.00 hours cortisol' and 'mean rest day hostility scores'. Since hostility and subservience are likely to show a

Table 1. Pearson correlation matrix for psychometric indices

\begin{tabular}{l|rrrr}
\hline & & & & \\
Depression & $0.851^{*}$ & & \\
Hostility & $0.866^{*}$ & $0.863^{*}$ & & \\
Libido & -0.262 & -0.443 & $-0.542^{*}$ & \\
Extraversion & -0.450 & $-0.606^{*}$ & $-0.581^{*}$ & 0.387 \\
\cline { 2 - 5 } & Anxiety & Depression & Hostility & Libido \\
*Significant at $P<0.05$. & & & \\
\hline
\end{tabular}


Table 2. Pearson product moment correlation matrix for psychometric and hormonal indices, in male marathon runners $(\mathrm{n}=11)$

\begin{tabular}{lccc}
\hline & Age & Hostility & Lie \\
Mean rest day cortisol & -0.40 & -0.08 & $0.75^{*}$ \\
Mean rest day cortisol range & -0.30 & -0.12 & $0.77^{*}$ \\
Mean rest day 08.00h cortisol & -0.30 & -0.05 & $0.85^{*}$ \\
Race day 08.00h cortisol & -0.71 & -0.53 & 0.35 \\
Mean rest day testosterone & -0.49 & -0.12 & 0.23 \\
Mean rest day testosterone range & -0.42 & -0.13 & 0.12 \\
Mean rest day 08.00h testosterone & -0.37 & -0.20 & 0.13 \\
Race day 08.00h testosterone & $-0.79 *$ & -0.19 & 0.56 \\
*Significant at $P<0.05$. & & & \\
\hline
\end{tabular}

Table 3. Spearman rank order correlation matrix for psychometric and hormonal indices in male marathon runners $(\mathrm{n}=11)$

\begin{tabular}{lcc}
\hline & Age & Hostility \\
Mean rest day cortisol & -0.39 & -0.31 \\
Mean rest day cortisol range & -0.07 & -0.47 \\
Mean rest day 08.00h cortisol & 0.09 & 0.04 \\
Race day 08.00h cortisol & $-0.67 *$ & $-0.66^{*}$ \\
Mean rest day testosterone & -0.40 & -0.36 \\
Mean rest day testosterone range & -0.46 & -0.15 \\
Mean rest day 08.00h testosterone & -0.47 & -0.26 \\
Race day 08.00h testosterone & $-0.81^{*}$ & -0.32 \\
*Significant at $P<0.05$. & & \\
\hline
\end{tabular}

negative correlation, these data imply a positive correlation of cortisol with subservience. Studies in monkeys, have also demonstrated such a positive correlation'. The score on the lie scale correlated positively with mean cortisol, cortisol range and mean $08.00 \mathrm{~h}$ cortisol on rest days. Since a high score on the lie scale indicates a tendency to give the expected and acceptable answer, this is a further indication of subservience. Thus, the correlation of the lie scale with cortisol also accords with the earlier findings of Keverne. ${ }^{9}$

While the $08.00 \mathrm{~h}$ levels of cortisol on the race day were negatively associated with age, other cortisol parameters showed no such correlation, which is in keeping with consensus opinion that adrenal activity is not age-dependent. In marked contrast, age was negatively associated with all parameters of testosterone and this correlation was highly significant for race day $08.00 \mathrm{~h}$ values. Negative correlation of salivary testosterone with age in an unselected population has been previously reported ${ }^{16}$. No correlation was found in this study between testosterone and libido. This is of interest since moderate running training has been previously associated with both reduced testosterone and, less convincingly, loss of libido ${ }^{17}$.

Whilst anxiety itself did not correlate with any cortisol parameter, in the volunteers who completed the psychological scales just prior to the marathon $(n=5)$, differences between $09.00 \mathrm{~h}$ control day and $09.00 \mathrm{~h}$ race day scores for anxiety were correlated $(r=0.6)$ with changes in cortisol at these times. In this smaller group, the correlation failed to achieve significance. These data are shown in Figure 2. Sutton and Casey also noted a significant increase in pre-race plasma cortisol compared with rest day values and presumed this was due to 'stress' ${ }^{\prime}$. Others conclude that plasma
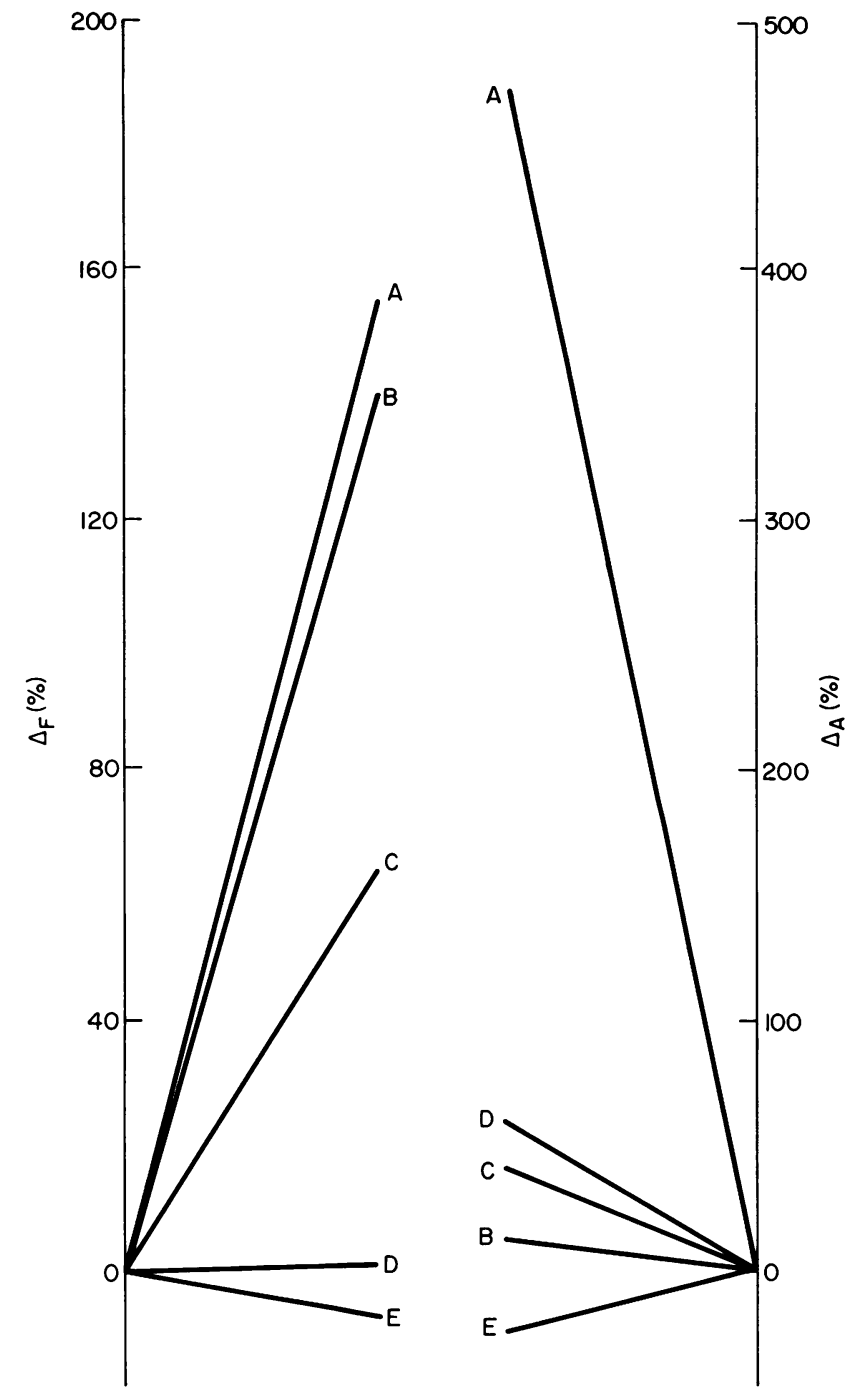

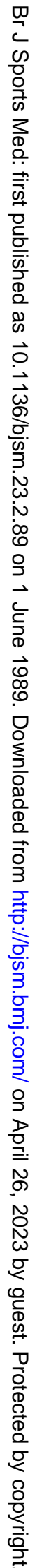

Figure 2. $\triangle F(\%)$ Change in cortisol between 09.00 on race day and mean level at 09.00 on three pre-race days, as a percentage of pre-race mean

$\triangle A(\%)$ As above, for anxiety

A-E Subjects

cortisol may increase before exercise but that this increase depends, in part, on fitness and training ${ }^{1 \overline{8}}$. The relationship between cortisol and anxiety reported in the present paper is essentially in keeping with those of Allen et al. who found no significant changes in hormone levels in the four to five week period preceding a stressful event (university examination) although anxiety, depression and distress increased ${ }^{19}$. On the day of the examination, however, mood changes were associated with significant increases in cortisol.

The preliminary data presented here suggest an interrelationship between psychological and hormonal parameters in marathon runners. The response of endocrine systems to mood change may be subtle and subject to some delay. Thus effective monitoring of response requires multiple sampling regimens, for which the frequent easy collection of saliva is an attractive method. The combination of simple self-rating psychological scales and measurement of salivary steroids appears to be well suited for future investigations of possible associations between mood and endocrine function. 


\section{References}

1 Wildmann, J., Kruger, A., Schmole, M., Niemann, J. and Matthaei, $\mathrm{H}$. Increase of circulating beta-endorphin-like immunoreactivity correlates with the change in feeling of pleasantness after running Life Sci 1986, 38, 997-1003

2 Greist, J.H., Klein, M.H., Eischens, R.R., Faris, J., Gurman, A.S. and Morgan, W.P. Running as treatment for depression Compr Psych 1979, 20, 41-54

3 Peters, J.R., Walker, R.F., Riad-Fahmy, D. and Hall, R. Salivary cortisol assays for assessing pituitary adrenal reserve Clin Endocrinol (Oxf) 1982, 17, 583-592

4 Walker, R.F., Wilson, D.W., Read, G.F. and RiadFahmy, D. Assessment of testicular function by the radioimmunoassay of testosterone in saliva Int J Androl $1980,3,105-120$

5 Semple, G.G., Thompson, J.A. and Beastall, G.H. Endocrine response to marathon running Brit J Sports Med 1985, 19, 148-151

6 Cook, N., Read, G.F., Walker, R.F., Harris, B. and Riad-Fahmy, D. Changes in adrenal and testicular activity monitored by salivary sampling in males throughout marathon runs Eur J Appl Physiol 1986, 55, 634-638

7 Cook, N., Read, G.F., Harris, B. and Riad-Fahmy, D. Salivary cortisol for monitoring adrenal activity during marathon runs Hormone Res 1987, 25, 18-23

8 Sutton, J.R. and Casey, J.H. The adrenocortical response to competitive athletics in veteran athletes $J$ Clin Endocrinol Metab 1975, 40, 135-138

9 Keverne, E.B., Meller, R.E. and Bernart, G.H. In 'Advanced views in primate biology' (Edited by Chiarelli A.B. and Curriccini R.S.) Berlin, Springer, 1982
Salivary steroids and psychometric parameters: B. Harris et al.

10 Jeffcoate, W.J., Lincoln, M.B., Selby, C. and Herbert, $M$. Correlation between anxiety and serum prolactin in humans J Psychosom Res 1986, 30, 217-222

11 Cook, N., Harris, B., Walker, R., Hailwood, R., Jones, E., Johns, S. and Riad-Fahmy, D. Clinical utility of the dexamethasone suppression test assessed by plasma and salivary cortisol determinations Psych Res 1986, 18, 143-150

12 Ekins, R.P. The precision profile: its use in RIA assessment and design Ligand Quarterly 1981, 4, 33-44

13 Luvia, R.E. The use of the visual analogue mood and alert scales in diagnosing hospitalised affective psychoses Psychol Med 1979, 9, 155-164

14 Alder, E. and Bancroft, S. Hormones, mood and sexuality in lactating women $\mathrm{Br} J$ Psychiat 1986, 148, 74-79

15 Eysenck, H.J. and Eysenck, B.G. 'Eysenck personality inventory' University of London Press, 1964

16 Read, G.F., Harper, M.E., Peeling, W.B. and Griffiths, $\mathrm{K}$. Changes in male salivary testosterone concentrations with age Int J Androl 1981, 4, 623-627

17 Wheeler, G.D., Wall, S.R., Belcastro, A.N. and Cumming, D.C. Reduced serum testosterone and prolactin levels in male distance runners JAMA 1984, 252, 514516

18 Dessypris, A., Kuoppasalmi, K. and Adlercreutz, H. Plasma cortisol, testosterone, androstenedione and luteinising hormone (LH) in a non-competitive marathon run J Steroid Biochem 1976, 7, 33-37

19 Allen, P.I.M., Batty, K.A., Dood, C.A.S., Herbert, J., Hugh, C.J., Moore, G.F., Seymour, M.J., Shiers, H.M., Stacey, P.M. and Young, S.K. Dissociation between emotional and endocrine responses preceding an academic examination in male medical students J Endocr 1985, 107, 163-170 